\author{
Jacques Henrard \\ Facultés Universitaires de Namur, B-5000 Namur, Belgium
}

The theory of the motion of the Moon can be approached by two types of methods. In the analytical methods, the solution is usually expanded in formal power series of the small quantities $m, e$ and $\gamma$. It is known that the expansions in $m$ are slowly convergent and that, in order to obtain an accuracy of the order of the meter, the expansions have to be carried to very high order (beyond 20) involving several hundred thousands terms.

In the numerical methods, the solution is sought under the form of a Fourier series solution (with about a thousand terms). The coefficients are determined by an iterative procedure.

While being conceptually more elegant (although its mathematical foundations are not firmer than those of the numerical methods), the analytical methods are wasteful. Many of the terms computed are numerically very small and the overall accuracy depends on a few very poorly convergent subseries. On the other hand, the fact that analytical methods provide a tube of trajectories rather than one particular solution is very important. With a single trajectory as usually provided by the numerical methods, it is impossible to improve on the solution by including other effects or by adjusting the constants.

It is thus natural to try to blend the advantages of the two types of techniques. Mrs. Chapront at the Bureau des Longitudes (Paris, France) is including in a numerical scheme the computation of the partial derivatives of the solution with respect to the constants of motion in order to get what one could call a semi-numerical solution. On the other hand, we are developping an analytical solution expanded not around the zero of the small parameter $m, e, \gamma$ but around their nominal values. In this way, at each order, the functions are no longer exact finite Fourier series but truncated Fourier series and only numerically meaningful terms are kept. We like to call this solution a semi-analytical solution.

With these two approaches, the gap between analytical and numerical 73

R. L. Duncombe (ed.), Dynamics of the Solar System, 73-75. Copyright $\odot 1979$ by the IAU. 
methods seems to be bridged. Nevertheless, there remains an important difference between the two philosophies. The difficult problem of resonance presents itself quite differently in order by order methods and in iterative methods. Much more work should be done before we can comment meaningfully on this difference.

\section{SEMI-ANALYTICAL METHOD}

We propose to base our solution on a completely analytical solution of Hill's Problem that we describe elsewhere ( $\mathrm{J}$. Henrard, Celestial Mechanics, in print). Hill's Problem is much simpler, so that a good analytical solution is feasible without too many problems. Our solution is complete up to order 25 and we estimate its accuracy at $1.10^{-10}$ in longitude.

This solution is given implicitely in functions of the angular variables

$$
\lambda=1+g+h \quad p=-g-h \quad q=-h
$$

and their conjugate momenta $L, P$ and $Q$ which, together with the initial values of the angular variables, are the constants of the motion.

If we introduce the canonical transformation

$$
L=L^{\star}(1+\Delta L) \quad P=P^{\star}+L^{\star} \Delta P \quad Q=Q^{\star}+L^{\star} \Delta Q
$$

from $(\lambda, p, q, L, P, Q)$ to $(\lambda, p, q, \Delta L, \Delta P, \Delta Q)$, we can express the solution of Hill's Problem and, by substitution, the perturbation function of the Main Problem in powers of $\Delta L, \Delta P$ and $\Delta Q$. The radius of convergence of these series appears to be around $1 / 200$ which is much larger than what we need. We choose to truncate them at degree 4 .

The coefficients in these expansions are Fourier series in the angular variables $\lambda, p$ and $q$. Of course, in order to manipulate these, we have to truncate them. We can allow the relative truncation error to be larger when the degree in $\Delta L, \Delta P, \Delta Q$ is higher, so that only a few terms are kept at degree $\overrightarrow{4}$ against several thousands at degree 1. A relative simple scheme determines the truncation level of each function.

With these new canonical variables, we can apply the Lie transform method and eliminate the periodic terms from the perturbation functions. We choose to eliminate separately the monthly terms, the annual terms and the long period terms. This is not imposed by the problem but, in this way, the analysis of the propagation of the truncation errors is much simplified. In the semi-analytical method we propose, this analysis is of prime importance and replaces in some way the analysis of the convergence of the subseries in the purely analytical method. 


\section{RESULTS FOR THE MAIN PROBLEM}

The elimination of the periodic terms from the hamiltonian function of the Main Problem and the expansion of the corresponding solution have been carried out on a computer.

According to our estimates, the solution is obtained with a relative accuracy of about $2.510^{-10}$ (or $5.10^{-5}$ in longitude) within a tube of solution corresponding to a relative change in eccentricity of $1.10^{-2}$. If we take into acount only the first partial derivatives, the same accuracy can be kept for a relative change in eccentricity of $1.10^{-5}$.

A comparison with the Analytical Lunar Ephemeris of Deprit, Henrard and Rom has been made and does not infirm our estimate of accuracy.

Our solution consists in the expansions of the three traditional functions : longitude, latitude and sine parallax, but also in the expansions of $r$ (the distance Earth-Moon) and $x / r, y / r$ and $\mathrm{z} / \mathrm{r}$ (the unit vector in the direction of the Moon). Together with the coefficients of the Fourier series, we give the first partial derivatives with respect to the internal constants ( $v$ - mean motion of the Moon -, E - coefficient of $\sin 1$ in the longitude -, $\Gamma$ coefficient of $\sin F$ in the latitude -) and the external constants ( $e_{2}$ - eccentricity of the Earth - , $\mu_{2}$ - mass ratio of the system Earth-Moon -, $\mu_{1}$ - mass ratio of the Moon -).

The solution is under a form directly suitable for the computation of further perturbations. Preliminary results have been obtained for the perturbation by the oblateness of the Earth by the author and for the direct perturbation of Venus by Daniel Standaert. 